\title{
Vocabulary Test for Vocabulary Learning in Middle School
}

\author{
Qilin Yan* \\ Jiangxi University of Technology, Nanchang 330098, China \\ *Corresponding author: Qilin Yan, yanqilin70@163.com
}

\begin{abstract}
As one of the core aspects and basic unit in language, vocabulary plays a salient role in improving student's language ability. Besides, vocabulary learning strategies play an obviously important part in the whole process of language learning. By examining the theories and approach to the vocabulary test, found by linguists locally and abroad, as well as vocabulary learning strategies, this research tries to systematically explicate several vocabulary tests in order to help English teachers design more creditable vocabulary tests to evaluate student's vocabulary knowledge. At the same time, in hope that through this research, middle school students would be guided to use English vocabulary learning strategies more efficiently, thus enhancing their self-learning ability.
\end{abstract}

Keywords: Middle school; Vocabulary test; Vocabulary learning

Publication date: August 2021; Online publication: August 30, 2021

\section{Introduction}

As is known to all, there are various tests, such as linguistic elements tests, linguistic skills tests, and even up-to-date tests for communicative competence. In particular, recently, more scholars and researchers have begun to center on the study of the vocabulary test. Vocabulary testing is now receiving the attention it deserves, with studies of the construct validity of several vocabulary tests, examination of the effectiveness of particular item types ${ }^{[1,2]}$, and the comprehensive examination of the field of vocabulary testing ${ }^{[3]}$. Although there are many achievements in the research on the vocabulary test, learners pay minimal attention to the study of the vocabulary test for students in specific levels, especially for beginners like students in middle school.

The study of the vocabulary test for students in middle school has much practical value. Firstly, it would help L2 learners at this level to acquire words efficiently, thus laying a good foundation for their future language learning. Secondly, it would be able to provide more reliability for teachers in order to gain sound knowledge of their vocabulary size or level and help them offer target ways in teaching. This paper focuses on vocabulary measuring instruments and compares different vocabulary test approaches while analyzing the features of L2 learners at the beginner level so as to determine efficient ways to acquire words.

\section{Vocabulary test}

The vocabulary test is generally divided into vocabulary levels test and vocabulary size test, in which the width, depth, as well as the productive and receptive vocabulary knowledge test are subdued. The lexical view is added as different views would lead to different test methods. 


\subsection{Vocabulary size test}

\subsubsection{Width of vocabulary}

The width of a word refers to how many meanings a word can have. Measuring the vocabulary size in isolation refers to the process of completing a word recognition test. Cameron resorted to this checklist test [4].

Example 1:
(a) $\square$ obey
(f) $\square$ accident
(b) $\square$ escape
(g) $\square$ common
(c) $\square$ graduate
(h) $\square$ shine
(d) $\square$ expect
(i) $\square$ sadly
(e) $\square$ large
(j) $\square$ check

Note: Tick the box(es) that belong(s) to the word(s) that you are familiar with.

A number of points can be made about the checklist format. A checklist test has no direct demonstration of knowledge, and there is always the chance that the examinees would overestimate their vocabulary knowledge; that is, checking the items that they do not know. In addition, the decontextualized presentation of a word in the test does not imply that the examinee makes sense of the test word in the decontextualized context.

\subsubsection{Depth of vocabulary}

The depth of vocabulary refers to how well words are known. This is useful because it indicates areas of weaknesses which deserve more attention in vocabulary development. There are three categories (form, meaning, and use), of which the category of "form" includes spoken, written, and word parts, "meaning" includes concept, referents, and associations, while "use" can be divided into three parts: grammatical functions, collocations, and constraints on use. Although Richards did not set out to create an all-inclusive description of vocabulary knowledge ${ }^{[5]}$, others have characterized it as such and have built upon it to create a more complete vocabulary knowledge framework. Perhaps, the most comprehensive descriptions of word knowledge were those proposed by Nation ${ }^{[6]}$, who listed the above-mentioned nine different specific types of knowledge that are necessary in order to know a word by revising them and adding another aspect "word parts."

\subsubsection{Productive vocabulary}

In the Productive Vocabulary Size Measures (also called PVLT), Laufer and Nation used the word frequency bands from the form-recognition version of the Vocabulary Levels Test (VLT) to create a formrecall version ${ }^{[2]}$.

\section{Example 2:}

(a) Every working person must pay income $t$

(b) The differences were so sl___ that they went unnoticed.

(c) There are a doz___ eggs in the basket.

Note: Examinees are required to fill in the blanks with the initial letters provided at the beginning of the blanks. 
This method changes the items on the test in regard to both their formal characteristics (number of letters) and the defining power of the context sentences as shown in Example 2, which lacked strong collocations and increased the difficulty of blank filling. However, it does not lay as much emphasis on the output of vocabulary as real productive mastery, but it is more likely to recall the link of form and meaning.

\subsection{Vocabulary Levels Test}

(1) Lexical frequency profile

The frequency counts used are the ones commonly available: words have been taken from the Thorndike and Lorge list by stratified sampling with reference to the frequency data from the GSL (General Service List). The only exception to this is the 2000 section, where words from the 1000word level and the 2000-word level have been sampled at a 1:2 ratio. The following illustrates the format of a noun cluster.

Example 3:

Sections of the Vocabulary Levels Test

2000-word level
(a) copy
( ) end or highest point
(b) events
(c) motor
( ) this moves a car
(d) 4 pities

Academic vocabulary
(a) area
( ) written agreement
(b) contract
(c) definition ( ) way of doing something
(d) evident

Note: Choose the right word to go with each meaning and write down the alphabet that belongs to that word next to its meaning.

(2) Dimensions (components) approach

It involves specifying some of the types of word knowledge one can have about lexical items, and then quantifying the learners' mastery of those types. The most outstanding potential advantage is its possible comprehensiveness. It can produce a rich description of vocabulary knowledge and a simplifying effect of breaking complex behavior into more manageable components for analysis. Nevertheless, it has limitations as well. In practical terms, it is impossible to measure all word knowledge aspects. Besides, researchers are unsure whether receptive and productive knowledge have a continuum, thus the shift of vocabulary from the receptive level to the productive level is not well understood.

\subsection{Lexical view}

\subsubsection{Lexical richness}

A measure of lexical richness should allow the language distinguishment between more proficient learners from less proficient ones. This can be done by analyzing the differences in the type-token ratio and the word frequency of items in texts. It can also be done in other ways, such as looking at the relationships between the words in a text and counting the total lexical errors. A measure of lexical richness should also be able to indicate the strengths and weaknesses of a learner's productive vocabulary. 
The type-token ratio is calculated using the following formula:

Type-token ratio $=$ number of different words (types) $/$ total number of different words in the text (tokens) $* 100$

The three examples below are all made up of four types and five tokens. However, the second and third examples consist of less frequent words, which may provide much more precise descriptions. The text length as well as the number of types and tokens are identical in each example, so measures of lexical variation would not differentiate between them.

Example 4:

(a) The man saw the woman.

(b) The bishop observed the actress.

(c) The magistrate sentenced the burglar.

Another issue is how to treat errors. If words are mispronounced, misspelled, or used incorrectly, should they be included in the measurements of lexical richness? Most studies lacked details in the way that errors are treated.

\subsubsection{Lexical organization}

(1) Test of lexical association (TLA)

One method is writing the first word which comes into one's mind when seeing a list of words. Another different approach to analyzing associations related to graph theory is to concentrate on the interconnectivity of the responses. A participant would be asked to indicate the strength of any connection he or she has selected, such as quiet-morning, quiet-peace, and quiet-sound. This method discriminates native speakers from non-native speakers and supports the notion that organization is a viable independent construct of vocabulary knowledge except size.

(2) Test of English derivatives (TED)

The aim of TED can be included in the test of lexical association. However, the test method is quite different.

Example 5: "philosophy"

Noun She explained her of life to me.

Verb She was known to about her life.

Adjective She was known as a ___ person.

Adverb She discovered her life

Note: According to the target word and the limited form provided, write appropriate derivatives in the blank spaces.

This can be informative as Schmitt and Zimmerman found that even relatively advanced learners (students studying professional courses and preparing to enter English-medium universities) often do not know the main derivatives of the target words in the Academic Word List ${ }^{[7]}$.

\section{Vocabulary test in middle school}

Multiple choice test is favored since it is simple to carry out and flexible to test many aspects of vocabulary knowledge. Apart from questionnaires to determine students' learning strategies, the vocabulary test 
volume should also be creditable by emphasizing different correlated parts.

\subsection{Test to correlate with students' cognitive strategy}

3.1.1. Receptive multiple-choice test in context (guessing, dictionary, and semantic or contextual encoding strategies)

Stewart used a multiple-choice vocabulary test and found that students still preferred learning vocabulary by rehearsing and note-taking whereas dictionary strategies and rehearsal strategies (listing out words or repeating words) were negatively related to vocabulary test scores ${ }^{[8]}$.

Example 6:

Single: She is still single, even if she is 35 years old now.

(a) unmarried

(b) only for one person

(c) not returned

Note: Choose the correct one, which has the same lexical meaning with the word in the given context.

When looking up in the dictionary, one can find the following sentences:

(a) She is still single, even if she is 35 years old now. (single $\rightarrow$ unmarried)

(b) Do you want to buy a single ticket or a return one? (single $\rightarrow$ not returned)

(c) Do you want to reserve a single or a double room? (single $\rightarrow$ only for one person)

Students might guess or eventually look up in the dictionary to select the correct answers or resort to encoding strategies. With the test results data collected, and an interview or questionnaire to aid the former, strategies used by students can be easily analyzed. For long-term learning benefits, the latter encoding strategies should be highlighted. Without a dictionary at hand and a large number of this type of test can ensure that those strategies will be emphasized.

\subsubsection{Vocabulary phonology test (visual and auditory encoding strategies)}

"It is often adopted among middle school students [9]." Hong drew this conclusion after conducting a research according to the "Learning Strategies Questionnaire," edited by Oxford and several English learning strategies among middle school students. Researchers have attached great importance to visual or auditory encoding strategy and proposed that the test of vocabulary phonology is also a good way to guide learners to acquire solid vocabulary knowledge, thus promoting their future learning of words.

\subsection{Test to emphasize social and affective strategies}

It deserves our attention to lay emphasis on social and affective strategies. Conversations or dialogues are good ways to test students' communication ability. The following test is one of the methods recommended to highlight or stimulate middle school students' social and affective strategies:

Example 7:

A: Hello! (1)---(b)---

B: Yes, what is it?

A: (2)---(c)--- Could I use your bike again?

B: Certainly. (3)---(a)--- Is your bike broken again?

A: No, I have lost my key for the bike. 
B: I see. (4)---(f)---

A: About four o'clock.

B: Okay. Here is the key (5)---(d)---

A: Thank you. Bye!

(a) What happened? (b) Is there anyone at home? (c) I am sorry to trouble you. (d) I hope everything is going well. (e) Be sure to be there on time. (f) What is the time? (g) Nice to meet you.

Note: Choose from the following items: (a) to (g) to fill in the blanks to complete the above dialogue.

A large number of such test with varying contextual conversation can ensure that the examinees are not using memory strategy but social or affective strategy.

\subsection{Test application in metacognitive strategy}

Metacognitive strategy is concerned with selective attention and self-initiation; thus, it is generally an internal or mental process. It is difficult to determine the learners' vocabulary learning strategies in a completely objective way. Nevertheless, it is essential to guide or stimulate learners to self-direct; hence, shifting the focus to student-oriented teaching. It would be better to take into account of each learner's cognitive ability and learning characteristic in order to use different levels of test or vocabulary learning tasks to stimulate their self-discovery and self-encouragement. This would lead them to self-direct with a bit of progress made each time and to avoid their fear of failing. It is self-evident that there is still a lot to be done in respect to this.

\section{Conclusion}

It can be concluded that the vocabulary test refers to various steps, methods, and techniques taken by teachers to enhance efficient vocabulary learning among students, and a good test should also test whether students have applied the aforementioned learning strategies. The test of vocabulary has accompanied the shift from teacher-oriented to learner-centered. The choice should depend on the extent of which the learning conditions might contribute to the aspects of knowledge.

The test of word formation should be highlighted so that students can apply the learning strategy of encoding root, prefix, and others similarly. Taking the term "bookseller" as an example, we can divide it into "book" and "seller." Students should use learning strategies to memorize it. The test of word formation can also determine whether students have used learning strategies, which can help improve their vocabulary learning.

Vocabulary size test should also be highlighted as the level for the lexical frequency profile will be very limited for beginners. Among the vocabulary size test, the depth of vocabulary should be strongly recommended, such as testing students' knowledge of lexical associations and derivatives.

\section{Funding}

This research was supported by 2019 Jiangxi Teaching Reform Project - The Teaching Mode Construction of Business English Reading Courses Based on PCT (JXJG-19-50-7) and 2020 Educational Reform and Research Project of Nanchang Institute of Technology - A Research of Ideological and Political Teaching Design and Practice against the New Background of Science and Engineering Courses (2020SZJG008).

\section{Disclosure statement}

The author declares that there is no conflict of interest. 


\section{References}

[1] Schmitt N, Nation P, Kremmel B, 2020, Moving the Field of Vocabulary Assessment Forward: The Need for More Rigorous Test Development and Validation. Language Teaching, 53(1): 109-20.

[2] Laufer B, Nation PA, 1995, Vocabulary-Size Test of Controlled Productive Ability. Language Testing, 16(1): 33-51.

[3] Read J, 2013, Validating a Test to Measure Depth of Vocabulary Knowledge, Validation in Language Assessment, Routledge, New York, 55-74.

[4] Cameron L, 2002, Measuring Vocabulary Size in English as an Additional Language. Language Teaching Research, 6(2): 145-73.

[5] Richards JC, 1976, The Role of Vocabulary Teaching. TESOL Quarterly, 10(1): 77-89.

[6] Nation P, 2001, Learning Vocabulary in Another Language, Cambridge University Press, Cambridge.

[7] Schmitt N, Zimmerman CB, 2002, Derivative Word Forms: What Do Learners Know?. TESOL Quarterly, (2): 145-71.

[8] Stewart J, 2012, A Multiple-Choice Test of Active Vocabulary Knowledge. Vocabulary Learning and Instruction, 1(1): 53-9.

[9] Hong X, 2007, A Study on the Developmental Characteristics of Middle School Students' English Vocabulary Memory Strategies. Journal of Leshan Normal University, (11): 130-3.

[10] Nation IS, Webb SA, 2011, Researching and Analyzing Vocabulary, Heinle Cengage Learning, Boston, MA. 